TP Periodica Polytechnica Mechanical Engineering

61(4), pp. 309-319, 2017

https://doi.org/10.3311/PPme.11254

Creative Commons Attribution (i)

RESEARCH ARTICLE

\section{An Elasticity Solution for Vibration Analysis of Laminated Plates with Functionally Graded Core Reinforced by Multi-walled Carbon Nanotubes}

\author{
Vahid Tahouneh $^{1^{*}}$
}

Received 12 July 2017; accepted after revision 14 September 2017

\begin{abstract}
In the present work, vibration characteristics of functionally graded (FG) sandwich rectangular plates reinforced by multiwalled carbon nanotubes (MWCNTS) resting on Pasternak foundation are presented. The response of the elastic medium is formulated by the Winkler/Pasternak model. Modified HalpinTsai equation is used to evaluate the Young's modulus of the MWCNT/epoxy composite samples by the incorporation of an orientation as well as an exponential shape factor in the equation. The mass density and Poisson's ratio of the MWCNT/phenolic composite are considered based on the rule of mixtures. The proposed sandwich rectangular plates have two opposite edges simply supported, while all possible combinations of free, simply supported and clamped boundary conditions are applied to the other two edges. The effects of two-parameter elastic foundation modulus, geometrical and material parameters together with the boundary conditions on the frequency parameters of the sandwich plates are investigated.
\end{abstract}

\section{Keywords}

multiwalled carbon nanotubes, vibration, sandwich plates, modified Halpin-Tsai equation, three-dimensional elasticity solution

\footnotetext{
${ }^{1}$ Young Researchers and Elite Club, Islamshahr Branch,

Islamic Azad University, Islamshahr, Iran

*Corresponding author, e-mail: vahid.tahouneh@ut.ac.ir
}

\section{Introduction}

Nowadays, the use of carbon nanotubes in polymer/carbon nanotube composites has attracted wide attention [1]. A high aspect ratio, low weight of CNTs and their extraordinary mechanical properties (strength and flexibility) provide the ultimate reinforcement for the next generation of extremely lightweight but highly elastic and very strong advanced composite materials. On the other hand, by using of the polymer/CNT composites in advanced composite materials, we can achieve structures with low weight, high strength and high stiffness in many structures of civil, mechanical and space engineering.

Several researches have recently investigated the elastic properties of multiwalled carbon nanotube (MWCNT) and their composites [2, 3]. Gojny et al. [4] focused on the evaluation of the different types of the CNTs applied, their influence on the mechanical properties of epoxy-based nanocomposites and the relevance of surface functionalization. Therefore, the study of the mechanical performance of CNT-based composites and the discovery of possible innovative applications has recently attracted the interest of many researchers.

Several researchers have reported that mechanical properties of polymeric matrices can be drastically increased $[5,6]$ by adding a few weight percent (wt\%) MWCNTs. Montazeri et al. [5] showed that modified Halpin-Tsai equation with exponential Aspect ratio can be used to model the experimental result of MWCNT composite samples. They also demonstrated that reduction in Aspect ratio $(\mathrm{L} / \mathrm{d})$ and nanotube length cause a decrease in aggregation and above $1.5 \mathrm{wt} \%$, nanotubes agglomerate causing a reduction in Young's modulus values. Thus, it is important to determine the effect Aspect ratio and arrangement of CNTs on the effective properties of carbon nanotube-reinforced composite (CNTRC). Yeh et al. [6] used the Halpin-Tsai equation to shows the effect of MWCNT shape factor (L/d) on the mechanical properties. They showed that the mechanical properties of nanocomposite samples with the higher shape factor $(\mathrm{L} / \mathrm{d})$ values were better than the ones with the lower shape factor. The reinforcement effect of MWCNTs with different aspect ratio in an epoxy matrix has been carried out by Martone et al. [7]. They showed that progressive reduction of 
the tubes effective aspect ratio occurs because of the increasing connectedness between tubes upon an increase in their concentration. Also they investigated on the effect of nanotube curvature on the average contacts number between tubes by means of the waviness that accounts for the deviation from the straight particles assumption.

In structural mechanics, one of the most popular semi-analytical methods is differential quadrature method (DQM) [8-12], remarkable success of which has been demonstrated by many researchers in vibration analysis of plates, shells, and beams. Liu and Liew [13], Liew and Liu [14] presented DQM for free vibration analysis of Mindlin isotropic circular and annular sector plates with various types of boundary conditions. A new version of the DQM was extended by Wang and Wang [15] to analyze the free vibration of thin circular sector plates with six combinations of boundary conditions. Liew et al. [16] employed DQM for free vibration analysis of moderately thick plates on Winkler foundation. Yas and Sobhani [17] achieved the natural frequencies of rectangular continuous grading fiber reinforced (CGFR) plates resting on elastic foundations; The CGFR plate was simply supported at the edges and was assumed to have an arbitrary variation of fiber volume fraction in the thickness direction. The results obtained indicated the advantages of using CGFR plate with graded fiber volume fractions over traditional discretely laminated plates. Matsunaga [18] analyzed the natural frequencies and buckling stresses of FG plates using a higher order shear deformation theory which are based on the through the thickness series expansion of the displacement components. Zhou et al. [19] used Ritz method to analyze the free-vibration characteristics of rectangular thick plates resting on elastic foundations. Matsunaga [20] investigated a two-dimensional, higher-order theory for analyzing the thick simply supported rectangular plates resting on elastic foundations. Tornabene and Viola [21, 22] investigated free vibration of three and four parameter functionally graded parabolic panels and shells of revolution. For the discretization of the system equations the Generalized Differential Quadrature (GDQ) method had been used. Numerical results concerning functionally graded parabolic panels and shells showed the influence of the three parameters of the power-law distribution on their mechanical behavior. The mathematical fundamental and recent developments of differential quadrature method as well as its major applications in engineering are discussed in detail in book by Shu [23]. One can compare DQM solution procedure with the other two widely used traditional methods for plate analysis, i.e., Rayleigh-Ritz method and FEM. The main difference between the DQM and the other methods is how the governing equations are discretized. In DQM, the governing equations and boundary conditions are directly discretized, and thus elements of stiffness and mass matrices are evaluated directly. But in Rayleigh-Ritz and FEMs, the weak form of the governing equations should be developed and the boundary conditions are satisfied in the weak form. Generally by doing so larger number of integrals with increasing amount of differentiation should be done to arrive at the element matrices. In addition, the number of degrees of freedom will be increased for an acceptable accuracy.

In comparison with research works on the free vibration or buckling analyses of FG structures [24-27], a few research works have been done about vibration analysis of sandwich structures. Kamarian et al. [28] studied vibration analysis of sandwich beams. The material properties of the FG nanocomposite sandwich beam were estimated using the Eshelby-Mori-Tanaka approach. Anderson [29] studied stress and displacement fields of sandwich panel consists of orthotropic face sheets bonded to an isotropic core with a Young's modulus that varied exponentially with respect to the transverse direction. Kashtalyan and Menshykova [30] considered elasticity analysis of sandwich panels with a functionally graded core subjected to transverse loading. The Young's modulus of the core was assumed to vary exponentially through the thickness. Li et al. [31] studied free vibrations of FGSW rectangular plates with simply supported and clamped edges. Zenkour [32, 33] presented a two-dimensional solution to study the bending, buckling and free vibration of simply supported FG ceramic-metal sandwich plates. Sharma and Marin [34] considered wave propagation in micropolar thermoelastic solid half space with distinct conductive and thermodynamic temperatures. Marin [35] obtained the existence and uniqueness of the generalized solutions for the boundary value problems in elasticity of dipolar materials with voids. Marin [36] studied harmonic vibrations in thermoelasticity of microstretch materials sing a toupin type measure associated with the corresponding steady-state vibration. Marin et al. [37] considered a theory of thermoelasticity constructed by taking into account the heat conduction in deformable bodies which depends on two temperatures. Only a few references can be found that consider the effect of MWCNTs on the vibrational behavior of structures [38]. Farid et al. [39] studied three-dimensional (3-D) temperature dependent free vibration analysis of FGM curved panels resting on two parameter elastic foundation by using a hybrid semi-analytic, DQM. Jam et al. [40] used the new version of Rule of mixture to show the effect of waviness of CNT on the vibrational behavior of nanocomposite cylindrical panel. They considered different waviness conditions with variable aspect ratio and they understood that the waviness have a significant effect on the natural frequency of nanocomposite cylindrical panel. Despite the aforementioned extensive research on the free vibration analysis of structures resting on elastic foundations, to the author's best knowledge, still very little work has been done for vibration analysis of FG-MWCNTs structures. The aim of this study is to fill this apparent gap in this area by providing the 3-D vibration analysis results for FG-MWCNT sandwich plates with power-law distribution of nanotube. The effective material properties of the FG-MWCNTs plates are estimated using 
a modified Halpin-Tsai equation. Also a parametric study is carried out to highlight the influence of MWCNTs volume fraction in the structure thickness, type of CNT distributions and geometrical parameters on vibration behavior of sandwich FG plates resting on two-parameter elastic foundations.

\section{Problem Description}

Consider a sandwich rectangular plate with length $a$, width $b$, and thickness $h$ which is as depicted in Fig. 1. The structure is supported by an elastic foundation with Winkler's (normal) and Pasternak's (shear) coefficients. The deformations defined with reference to a Cartesian coordinate system $(x, y, z)$ are $u, v$ and $w$ in the $x, y$ and $z$ directions, respectively.

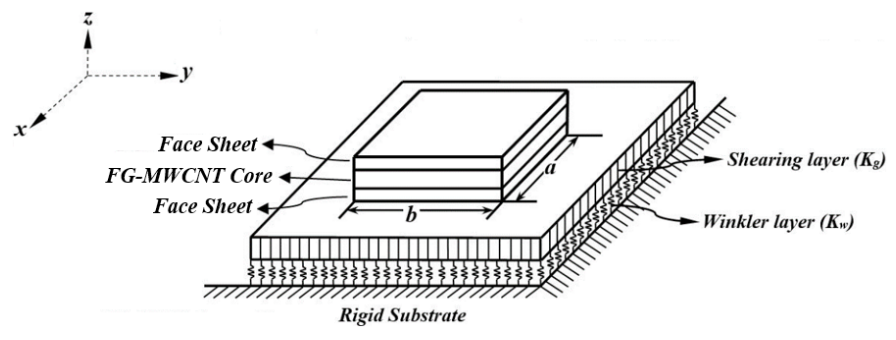

Fig. 1 Geometry and coordinates of the thick sandwich rectangular plate with FG-MWCNT Core and Face Sheets on an elastic foundation (the origin is placed in the middle of sandwich plate).

The structure has continuous grading of reinforcement through thickness direction. In this study, we will discuss about the results in the literature on mechanical properties of polymer nanotube composites. The Halpin-Tsai equation assumes that the filler is straight and uniform dispersion of the filler in the polymer matrix. The Halpin-Tsai equation [41, 42] has been recognized for its ability to predict the modulus values for the fiber-reinforced composite samples. The effective mechanical properties of the CNTRC plate are obtained based on a modified Halpin-Tsai equation according to $[5,6]$ :

$$
E=E_{m} \frac{1+\eta_{L} \eta_{T} V_{c n}}{1-\eta_{T} V_{c n}}, \eta_{T}=\frac{\alpha E_{c n} / E_{m}-1}{\alpha E_{c n} / E_{m}+\eta_{L}}
$$

The effective Young's modulus of MWCNT can be deduced from Eq. (1) as follows:

$$
E_{f}=\frac{\left(2 l / d+V_{c n}\right) E-2 l / d\left(1-V_{c n}\right) E_{m}}{a\left[\left(2 l / d+V_{c n}+1\right) E_{m}-\left(1-V_{c n}\right) E\right]} E_{m}
$$

From the linear region of the fitting line for MWNTs/phenolic composites, the effective Young's modulus $\left(E_{f}\right)$ of MWNT is $953 \mathrm{GPa}$. In above equations, $E_{c n}$ and $E_{m}$ are the longitudinal elastic moduli of the MWCNT and pure polymer; $V_{c n}$ is the CNT volume fraction; $\eta_{L}$ is the exponential shape factor; 1 and $\mathrm{d}$ are the length and the diameter of CNT and $\alpha$ is CNT orientation efficiency.

$$
\eta_{L}=2 \frac{l}{d} e^{-a V_{c n}-b}
$$

In which $\eta_{L}$ is related to the aspect ratio of reinforcement length 1 and diameter $d$ in the Halpin-Tsai equation. $a$ and $b$ are constants, related to the degree of MWCNTs aggregation, which account for the nonlinear behavior of the HalpinTsaai equation in the MWNTs wt $\%$ range considered $[5,6]$. The resulting effective properties for the randomly oriented MWCNT composite are isotropic, despite the CNTs having transversely isotropic effective properties. The orientation of a straight CNT is characterized by $\alpha$. When CNTs are completely randomly oriented in the matrix, the composite is then isotropic. In this article, the experimental data for the Young's modulus of MWCNT/phenolic composites with different mass fraction of MWCNTs, reported by Yeh et al. [6], was used to fit the above Halpin-Tsai. In Fig. 2, the predicted Young's moduli using Eq. (1) is shown. The best fit was achieved by taking the model parameters given in Table 1. Using this prediction model, the Young's modulus of functionally graded MWCNT/phenolic composites will be estimated during the numerical solutions in the next sections. Also, the mass density and Poisson's ratio of the MWCNT/phenolic composite according to rule of mixtures can be calculated, respectively, by

$$
\begin{aligned}
v_{i j} & =V_{c n} v^{c n}+V_{m} v^{m}, i j=12,13,23 \\
\rho & =V_{c n} \rho^{c n}+V_{m} \rho^{m}
\end{aligned}
$$

where $v^{c n}$ and $\rho^{c n}$ are Poisson's ratio and density, respectively, of the CNT and $v^{m}$ and $\rho^{m}$ are corresponding properties for the matrix. It is assumed that the following specific power-law variation of the reinforcement volume fraction, dictates the material profiles in the thickness of the sandwich plate [43]:

$$
V_{\mathrm{MWCNT}}= \begin{cases}V_{i} & -0.5 h \leq z \leq-0.5 h+h_{f} \\ V_{i}+\left(V_{o}-V_{i}\right)\left(\frac{z}{h}\right)^{p} & -0.5 h+h_{f} \leq z \leq 0.5 h-h_{f} \\ V_{o} & 0.5 h-h_{f} \leq z \leq 0.5 h\end{cases}
$$

where $V_{i}$ and $V_{o}$, which have values that range from 0 to 1 . The exponent "p" governs the through-thickness fiber volume fraction profile. The through-thickness variations of the volume fractions are depicted in Fig. 3. As shown in Fig. 3, the volume fraction of core varies from 0.2 to 0.8 as $\eta(\eta=z / h)$ varies from $-h_{c} / 2$ and $h_{c} / 2$ while the reinforcement volume fractions of top and bottom faces are 0.8 and 0.2 , respectively.

Table 1 Material properties for pure phenolic and the MWCNTs

\begin{tabular}{ll}
\hline Polymer (phenolic) & MWCNT \\
\hline$E_{m}=5.13 \mathrm{GPa}$ & $E_{c n}=953 \mathrm{GPa}, \rho_{c n}=1.3 \mathrm{~g} / \mathrm{ml}$, \\
$v_{c n}=0.29$ \\
$\rho_{m}=1.03 \mathrm{~g} / \mathrm{ml}$ & $\alpha=1 / 6, l=17.57 \mu \mathrm{m}$, \\
& $d=23.63 \mathrm{~nm}$ \\
$v_{m}=0.34$ & $a=75, b=1$ \\
\hline
\end{tabular}




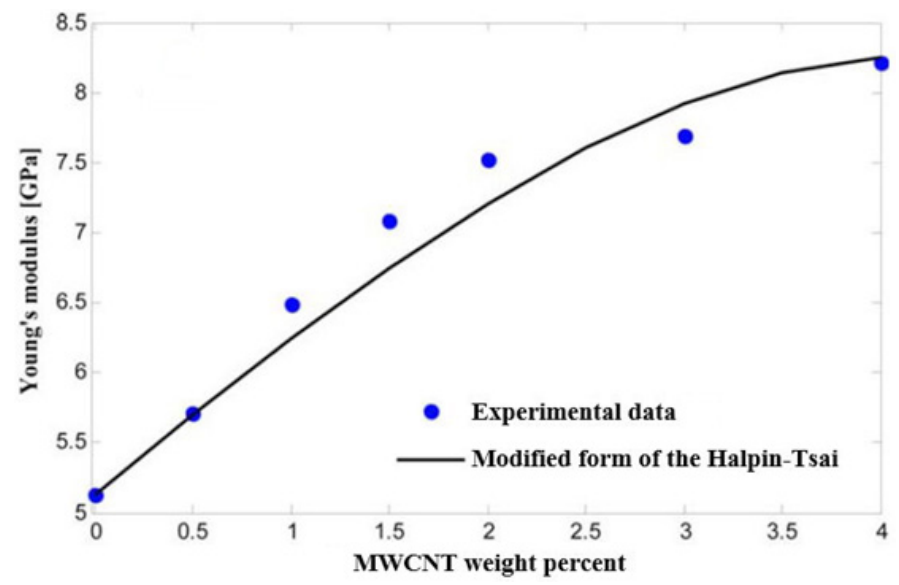

Fig. 2 Prediction of the Young's modulus of MWCNT/phenolic composites containing various $\mathrm{wt} \%$ of MWCNTs.

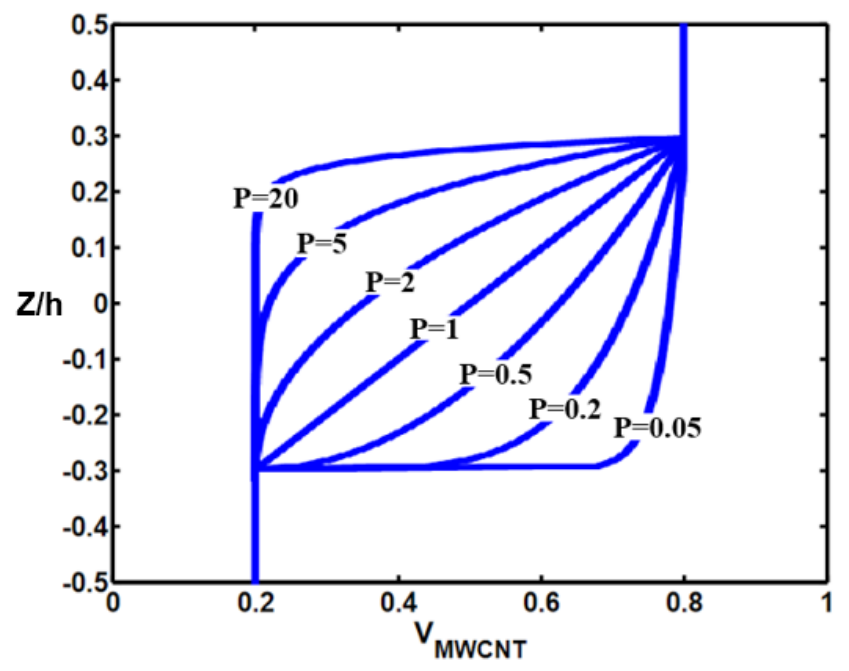

Fig. 3 Variations of the volume fraction of reinforcement $\left(\mathrm{V}_{\mathrm{MWCNT}}\right)$ in the thickness direction of sandwich rectangular plate for different values of "p".

\section{Governing equations}

The mechanical constitutive relations that relate the stresses to the strains are as follows

$$
\sigma_{i j}=\lambda \varepsilon_{k k} \delta_{i j}+2 \psi \varepsilon_{i j}
$$

where $\lambda$ and $\psi$ are the Lame constants, $\varepsilon_{i j}$ is the infinitesimal strain tensor and $\delta_{i j}$ is the Kronecker delta. In the absence of body forces, the equations of motion are as follows

$$
\begin{aligned}
& \frac{\partial \sigma_{x}}{\partial x}+\frac{\partial \tau_{x y}}{\partial y}+\frac{\partial \tau_{x z}}{\partial z}=\rho \frac{\partial^{2} u}{\partial t^{2}} \\
& \frac{\partial \tau_{x y}}{\partial x}+\frac{\partial \sigma_{y}}{\partial y}+\frac{\partial \tau_{y z}}{\partial z}=\rho \frac{\partial^{2} v}{\partial t^{2}} \\
& \frac{\partial \tau_{x z}}{\partial x}+\frac{\partial \tau_{y z}}{\partial y}+\frac{\partial \sigma_{z}}{\partial z}=\rho \frac{\partial^{2} w}{\partial t^{2}}
\end{aligned}
$$

the infinitesimal strain tensor is related to the displacements as follows

$$
\begin{aligned}
\varepsilon_{x} & =\frac{\partial u}{\partial x}, \varepsilon_{y}=\frac{\partial v}{\partial y}, \varepsilon_{z}=\frac{\partial w}{\partial z}, \gamma_{x y}=\frac{\partial u}{\partial y}+\frac{\partial v}{\partial x} \\
\gamma_{x z} & =\frac{\partial u}{\partial z}+\frac{\partial w}{\partial x}, \gamma_{y z}=\frac{\partial v}{\partial z}+\frac{\partial w}{\partial y}
\end{aligned}
$$

where $u, v$ and $w$ are displacement components along the $x$, $y$ and $z$ axes, respectively. Upon substitution (8) into (6) and then into (7), the equations of motion in terms of displacement components with infinitesimal deformations can be written as:

$$
\left[\begin{array}{ccc}
F_{1 x} & F_{1 y} & F_{1 z} \\
F_{2 x} & F_{2 y} & F_{2 z} \\
F_{3 x} & F_{3 y} & F_{3 z}
\end{array}\right]\left\{\begin{array}{l}
u \\
v \\
w
\end{array}\right\}=\left\{\begin{array}{c}
\rho \frac{\partial^{2} u}{\partial t^{2}} \\
\rho \frac{\partial^{2} v}{\partial t^{2}} \\
\rho \frac{\partial^{2} w}{\partial t^{2}}
\end{array}\right\}
$$

where coefficients $F_{i j}$ are given in Appendix. The related boundary conditions at $z=-h / 2$ and $h / 2$ are as follows:

$$
\begin{aligned}
& \text { at } z=-h / 2: \\
& \sigma_{z x}=0, \\
& \sigma_{z y}=0, \sigma_{z z}=K_{w} w-K_{g}\left(\frac{\partial^{2} w}{\partial x^{2}}+\frac{\partial^{2} w}{\partial y^{2}}\right) \\
& \text { at } z=h / 2: \\
& \sigma_{z x}=0, \\
& \sigma_{z y}=0, \\
& \sigma_{z z}=0
\end{aligned}
$$

where $\sigma_{i j}$ are the components of stress tensor; $K_{w}$ and $K_{g}$ are Winkler and shearing layer elastic coefficients of the foundation.

The stress components are related to the displacement components using the three-dimensional constitutive relations as

$$
\begin{aligned}
& \sigma_{x}=c_{11} \frac{\partial u}{\partial x}+c_{12} \frac{\partial v}{\partial y}+c_{13} \frac{\partial w}{\partial z}, \sigma_{y z}=c_{44}\left(\frac{\partial w}{\partial y}+\frac{\partial v}{\partial z}\right) \\
& \sigma_{y}=c_{12} \frac{\partial u}{\partial x}+c_{22} \frac{\partial v}{\partial y}+c_{23} \frac{\partial w}{\partial z}, \sigma_{x z}=c_{55}\left(\frac{\partial u}{\partial z}+\frac{\partial w}{\partial x}\right) \\
& \sigma_{z}=c_{13} \frac{\partial u}{\partial x}+c_{23} \frac{\partial v}{\partial y}+c_{33} \frac{\partial w}{\partial z}, \sigma_{x y}=c_{66}\left(\frac{\partial v}{\partial x}+\frac{\partial u}{\partial y}\right)
\end{aligned}
$$

Different types of classical boundary conditions at the edges of the plate can be stated as

- Simply supported (S):

$$
\sigma_{y y}=0, \quad w=0, u=0
$$

- Clamped (C):

$$
u=0, v=0, w=0
$$

- Free (F):

$$
\sigma_{y y}=0, \sigma_{x y}=0, \sigma_{y z}=0
$$


Here, plates with two opposite edges at $x=-a / 2$ and $a / 2$ simply supported and arbitrary conditions at edges $y=-b / 2$ and $b / 2$ are considered. For free vibration analysis, by adopting the following form for the displacement components the boundary conditions at edges $\mathrm{x}=-a / 2$ and $a / 2$ are satisfied,

$$
\begin{aligned}
& u(x, y, z, t)=U_{m}(y, z, t) \cos \left(m \pi(x+a / 2 / a) e^{i \omega t},\right. \\
& v(x, y, z, t)=V_{m}(y, z, t) \sin \left(m \pi(x+a / 2 / a) e^{i \omega t},\right. \\
& w(x, y, z, t)=W_{m}(y, z, t) \sin \left(m \pi(x+a / 2 / a) e^{i \omega t}\right.
\end{aligned}
$$

where $m$ is the wave number along the $\mathrm{x}$ - direction, $\omega$ is the natural frequency and $i(=\sqrt{-1})$ is the imaginary number. Substituting for displacement components from (16) into the equations of motion which obtained in terms of displacement components, the coupled partial differential equations are reduced to a set of coupled ordinary differential equations (ODE). The geometrical and natural boundary can also be simplified, however, for brevity purpose, they are not shown here.

It is necessary to develop appropriate methods to investigate the mechanical responses of functionally graded (FG) nanocomposite sandwich plates. But, due to the complexity of the problem caused by the inhomogeneity, it is difficult to obtain the exact solution. In this paper, the generalized differential quadrature method (GDQM) approach is used to solve the governing equations of rectangular plates.

Substituting for displacement components from (16) into (9), and then using GDQ method to discretize the equations of motion, one can get the following equations (A brief review of GDQ method is given in Ref. [23]):

In the $\mathrm{x}$-direction:

$-\left(c_{11}\right)_{j k}\left(\frac{m \pi}{a}\right)^{2} U_{m j k}+\left(c_{12}\right)_{j k}\left(\frac{m \pi}{a}\right) \sum_{n=1}^{N_{y}} A_{j n}^{y} V_{m n k}+\left(c_{13}\right)_{j k}\left(\frac{m \pi}{a}\right)$

$\sum_{n=1}^{N_{z}} A_{k n}^{z} W_{m j n}+\left(c_{66}\right)_{j k}\left(\frac{m \pi}{a} \sum_{n=1}^{N_{y}} A_{j n}^{y} V_{m n k}+\sum_{n=1}^{N_{y}} B_{j n}^{y} U_{m n k}\right)+\left(\frac{\partial c_{55}}{\partial z}\right)_{j k}$

$\left(\frac{m \pi}{a} W_{m j k}+\sum_{n=1}^{N_{z}} A_{k n}^{z} U_{m j n}\right)+\left(c_{55}\right)_{j k}\left(\frac{m \pi}{a} \sum_{n=1}^{N_{z}} A_{k n}^{z} W_{m j n}+\sum_{n=1}^{N_{z}} B_{k n}^{z} U_{m j n}\right)$

$=-\rho_{j k} \omega^{2} U_{m j k}$

In the y-direction:

$$
\begin{aligned}
& \left(c_{66}\right)_{j k}\left(-\left(\frac{m \pi}{a}\right)^{2} V_{m j k}+\left(\frac{-m \pi}{a}\right) \sum_{n=1}^{N_{y}} A_{j n}^{y} U_{m n k}\right)+\left(c_{12}\right)_{j k} \\
& \left(\left(\frac{-m \pi}{a}\right) \sum_{n=1}^{N_{y}} A_{j n}^{y} U_{m n k}\right)+\left(c_{22}\right)_{j k} \sum_{n=1}^{N_{y}} B_{j n}^{y} V_{m n k}+\left(c_{23}\right)_{j k} \\
& \left(\sum_{n=1}^{N_{y}} \sum_{r=1}^{N_{z}} A_{k r}^{z} A_{j n}^{y} W_{m n r}\right)+\left(\frac{\partial c_{44}}{\partial z}\right)_{j k}\left(\sum_{n=1}^{N_{z}} A_{k n}^{z} V_{m j n}+\sum_{n=1}^{N_{y}} A_{j n}^{y} W_{m n k}\right) \\
& +\left(c_{44}\right)_{j k}\left(\sum_{n=1}^{N_{z}} B_{k n}^{z} V_{m j n}+\sum_{n=1}^{N_{y}} \sum_{r=1}^{N_{z}} A_{k r}^{z} A_{j n}^{y} W_{m n r}\right) \\
& =-\rho_{j k} \omega^{2} V_{m j k}
\end{aligned}
$$

In the z-direction:

$$
\begin{aligned}
& \left(c_{55}\right)_{j k}\left(-\left(\frac{m \pi}{a}\right)^{2} W_{m j k}-\frac{m \pi}{a} \sum_{n=1}^{N_{z}} A_{k n}^{z} U_{m j n}\right) \\
& +\left(c_{44}\right)_{j k}\left(\sum_{n=1}^{N_{y}} \sum_{r=1}^{N_{z}} A_{k r}^{z} A_{j n}^{y} V_{m n r}+\sum_{n=1}^{N_{y}} B_{j n}^{y} W_{m n k}\right) \\
& +\left(\frac{\partial c_{13}}{\partial z}\right)_{j k}\left(-\frac{m \pi}{a} U_{m j k}\right)+\left(c_{13}\right)_{j k}\left(-\frac{m \pi}{a} \sum_{n=1}^{N_{z}} A_{k n}^{z} U_{m j n}\right)+\left(\frac{\partial c_{23}}{\partial z}\right)_{j k} \\
& \sum_{n=1}^{N_{y}} A_{j n}^{y} V_{m n k}+\left(c_{23}\right)_{j k} \sum_{n=1}^{N_{y}} \sum_{r=1}^{N_{z}} A_{k r}^{z} A_{j n}^{y} V_{m n r}+\left(\frac{\partial c_{33}}{\partial z}\right)_{j k} \sum_{n=1}^{N_{z}} A_{k n}^{z} W_{m j n}+\left(c_{33}\right)_{j k} \\
& \sum_{n=1}^{N_{z}} B_{k n}^{z} W_{m j n}=-\rho_{j k} \omega^{2} W_{m j k}
\end{aligned}
$$

where $A_{i j}^{y}, A_{i j}^{z}$ and $B_{i j}^{y}, B_{i j}^{z}$ are the first and second order DQ weighting coefficients in the $y$ - and $z$-directions, respectively. In a similar manner the boundary conditions can be discretized. In order to carry out the eigenvalue analysis, the domain and boundary nodal displacements should be separated. In vector forms, they are denoted as $\{d\}$ and $\{b\}$, respectively. Based on this definition, the discretized form of the equations of motion and the related boundary conditions can be represented in the matrix form as equations of motion:

$$
\left[\left[K_{d b}\right]\left[K_{d d}\right]\right]\left\{\begin{array}{l}
\{b\} \\
\{d\}
\end{array}\right\}-\omega^{2}[M]\{d\}=\{0\}
$$

and boundary conditions:

$$
\left[K_{b d}\right]\{d\}+\left[K_{b b}\right]\{b\}=\{0\}
$$

eliminating the boundary degrees of freedom in (20) using (21), this equation becomes,

$$
[K]-\omega^{2}[M]\{d\}=\{0\}
$$

where $[K]=\left[K_{d d}\right]-\left[K_{d b}\right]\left[K_{b b}\right]^{-1}\left[K_{b d}\right]$. The above eigenvalue system of equations can be solved to find the natural frequencies and mode shapes of the plates.

\section{Numerical results and discussion}

Firstly, the results are compared with those conventional functionally graded rectangular plates, and then, the results of the presented formulations are given in the form of convergence studies with respect to $N_{z}$ and $N_{y}$, the number of discrete points distributed along the thickness and width of the plate, respectively. The boundary conditions of the plate are specified by the letter symbols, for example, $S-C-S-F$ denotes a plate with edges $\mathrm{x}=-a / 2$ and $a / 2$ simply supported $(S)$, edge $\mathrm{y}=-b / 2$ clamped $(C)$ and edge $\mathrm{y}=b / 2$ free $(F)$.

As a first example, the properties of the plate are assumed to vary through the thickness of the plate with a desired variation of the volume fractions of the two materials in between the two surfaces. The modulus of elasticity $E$ and mass density $\rho$ are assumed to be in terms of a simple power- law distribution and Poisson's ratio $v$ is assumed to be constant as follows: 
Table 2 Convergence behavior and accuracy of the first seven non-dimensional natural frequencies $\left(\varpi=\omega h \sqrt{\rho_{C} / E_{C}}\right)$ of a simply supported FG plate against the number of DQ grid points $(b / h=2)$.

\begin{tabular}{|c|c|c|c|c|c|c|c|c|c|}
\hline$P$ & $N_{z}$ & $N_{y}$ & $\varpi_{1}$ & $\varpi_{2}$ & $\varpi_{3}$ & $\varpi_{4}$ & $\varpi_{5}$ & $\varpi_{6}$ & $\varpi_{7}$ \\
\hline \multirow[t]{11}{*}{0} & 7 & 7 & 0.5569 & 0.9395 & 0.9735 & 1.3764 & 1.5072 & 1.6064 & 1.7384 \\
\hline & & 9 & 0.5570 & 0.9396 & 0.9741 & 1.3771 & 1.5083 & 1.6071 & 1.7401 \\
\hline & & 13 & 0.5570 & 0.9396 & 0.9740 & 1.3774 & 1.5088 & 1.6076 & 1.7407 \\
\hline & 9 & 7 & 0.5573 & 0.9398 & 0.9735 & 1.3771 & 1.5087 & 1.6074 & 1.7403 \\
\hline & & 9 & 0.5572 & 0.9400 & 0.9742 & 1.3777 & 1.5090 & 1.6079 & 1.7406 \\
\hline & & 13 & 0.5572 & 0.9400 & 0.9741 & 1.3778 & 1.5096 & 1.6086 & 1.7405 \\
\hline & 13 & 7 & 0.5571 & 0.9401 & 0.9735 & 1.3779 & 1.5094 & 1.6083 & 1.7411 \\
\hline & & 9 & 0.5572 & 0.9400 & 0.9742 & 1.3777 & 1.5090 & 1.6078 & 1.7405 \\
\hline & & 13 & 0.5572 & 0.9400 & 0.9742 & 1.3777 & 1.5090 & 1.6078 & 1.7406 \\
\hline & & [18] & 0.5572 & 0.9400 & 0.9742 & 1.3777 & 1.5090 & 1.6078 & 1.7406 \\
\hline & & [17] & 0.557243 & 0.940041 & - & - & 1.508987 & - & 1.740602 \\
\hline \multirow[t]{11}{*}{0.5} & 7 & 7 & 0.4829 & 0.8222 & 0.8700 & 1.2250 & 1.3332 & 1.4364 & 1.5401 \\
\hline & & 9 & 0.4828 & 0.8229 & 0.8707 & 1.2258 & 1.3337 & 1.4367 & 1.5429 \\
\hline & & 13 & 0.4830 & 0.8224 & 0.8706 & 1.2254 & 1.3338 & 1.4370 & 1.5424 \\
\hline & 9 & 7 & 0.4833 & 0.8225 & 0.8701 & 1.2251 & 1.3335 & 1.4365 & 1.5402 \\
\hline & & 9 & 0.4835 & 0.8240 & 0.8708 & 1.2257 & 1.3340 & 1.4370 & 1.5431 \\
\hline & & 13 & 0.4836 & 0.8233 & 0.8707 & 1.2258 & 1.3340 & 1.4369 & 1.5426 \\
\hline & 13 & 7 & 0.4836 & 0.8227 & 0.8701 & 1.2251 & 1.3334 & 1.4366 & 1.5402 \\
\hline & & 9 & 0.4835 & 0.8231 & 0.8708 & 1.2259 & 1.3338 & 1.4370 & 1.5431 \\
\hline & & 13 & 0.4835 & 0.8233 & 0.8709 & 1.2259 & 1.3339 & 1.4370 & 1.5425 \\
\hline & & [18] & 0.4835 & 0.8233 & 0.8709 & 1.2259 & 1.3339 & 1.4370 & 1.5425 \\
\hline & & [17] & 0.482849 & 0.822358 & - & - & 1.332605 & - & 1.541085 \\
\hline \multirow[t]{11}{*}{1} & 7 & 7 & 0.4367 & 0.7476 & 0.7997 & 1.1158 & 1.2154 & 1.3085 & 1.4059 \\
\hline & & 9 & 0.4374 & 0.7477 & 0.8001 & 1.1165 & 1.2159 & 1.3090 & 1.4075 \\
\hline & & 13 & 0.4373 & 0.7478 & 0.8005 & 1.1163 & 1.2162 & 1.3088 & 1.4077 \\
\hline & 9 & 7 & 0.4368 & 0.7477 & 0.7998 & 1.1159 & 1.2157 & 1.3088 & 1.4068 \\
\hline & & 9 & 0.4374 & 0.7477 & 0.8003 & 1.1165 & 1.2161 & 1.3090 & 1.4076 \\
\hline & & 13 & 0.4374 & 0.7478 & 0.8006 & 1.1165 & 1.2162 & 1.3090 & 1.4078 \\
\hline & 13 & 7 & 0.4368 & 0.7477 & 0.7999 & 1.1159 & 1.2158 & 1.3088 & 1.4070 \\
\hline & & 9 & 0.4375 & 0.7478 & 0.8003 & 1.1165 & 1.2162 & 1.3091 & 1.4076 \\
\hline & & 13 & 0.4375 & 0.7478 & 0.8005 & 1.1165 & 1.2163 & 1.3091 & 1.4077 \\
\hline & & [18] & 0.4375 & 0.7477 & 0.8005 & 1.1166 & 1.2163 & 1.3091 & 1.4078 \\
\hline & & [17] & 0.437396 & 0.747514 & - & - & 1.216035 & - & 1.407459 \\
\hline \multirow[t]{11}{*}{4} & 7 & 7 & 0.3565 & 0.5988 & 0.6249 & 0.8724 & 0.9589 & 1.0000 & 1.1029 \\
\hline & & 9 & 0.3577 & 0.5995 & 0.6355 & 0.8729 & 0.9589 & 1.0007 & 1.1038 \\
\hline & & 13 & 0.3577 & 0.5996 & 0.6349 & 0.8728 & 0.9589 & 1.0003 & 1.1030 \\
\hline & 9 & 7 & 0.3569 & 0.5989 & 0.6250 & 0.8726 & 0.9589 & 1.0001 & 1.1032 \\
\hline & & 9 & 0.3579 & 0.5997 & 0.6357 & 0.8731 & 0.9589 & 1.0008 & 1.1040 \\
\hline & & 13 & 0.3578 & 0.5997 & 0.6351 & 0.8730 & 0.9589 & 1.0005 & 1.1032 \\
\hline & 13 & 7 & 0.3571 & 0.5991 & 0.6252 & 0.8727 & 0.9589 & 1.0001 & 1.1033 \\
\hline & & 9 & 0.3579 & 0.5997 & 0.6357 & 0.8731 & 0.9589 & 1.0008 & 1.1040 \\
\hline & & 13 & 0.3579 & 0.5997 & 0.6352 & 0.8731 & 0.9589 & 1.0008 & 1.1040 \\
\hline & & [18] & 0.3579 & 0.5997 & 0.6352 & 0.8731 & 0.9591 & 1.0008 & 1.1040 \\
\hline & & [17] & 0.357758 & 0.599494 & - & - & 0.958764 & - & 1.103674 \\
\hline
\end{tabular}




$$
\begin{aligned}
& E(z)=E_{M}+E_{C M} V_{f}, v(z)=v_{0}, \rho(z)=\rho_{M}+\rho_{C M} V_{f}, \\
& E_{C M}=E_{C}-E_{M}, \rho_{C M}=\rho_{C}-\rho_{M}, V_{f}=(0.5+z / h)^{p}
\end{aligned}
$$

where $-h / 2 \leq z \leq h$ and $p$ is the power-law index which takes values greater than or equal to zero. Subscripts $M$ and $C$ refer to the metal and ceramic constituents which denote the material properties of the bottom and top surface of the plate, respectively.

The mechanical properties are as follows:

- Metal (Aluminum, Al):

$$
E_{M}=70 * 10^{9} \mathrm{~N} / \mathrm{m}^{2}, v=0.3, \rho_{M}=2702 \mathrm{~kg} / \mathrm{m}^{3} .
$$

- Ceramic (Alumina, $\mathrm{Al}_{2} \mathrm{O}_{3}$ ):

$$
E_{C}=380 * 10^{9} \mathrm{~N} / \mathrm{m}^{2}, v=0.3, \rho_{C}=3800 \mathrm{~kg} / \mathrm{m}^{3} .
$$

In Table 2, the first seven non-dimensional natural frequency parameters of simply supported thick FG plate are compared with those of Matsunaga [18] and Yas and Sobhani [17].

As the second example, in order to validate the results for plates on an elastic foundation, the results for the first three natural frequency parameters of isotropic thick plate with two different values of thickness-to-length ratios and different values of Winkler elastic coefficient are presented in Table 3. They are compared with those of Zhou et al. [19], Matsunaga [20] and Yas and Sobhani [17]. In this example the non-dimensional natural frequency, Winkler and shearing layer elastic coefficients are as follows:

$$
\begin{aligned}
& \lambda=\omega \frac{b^{2}}{\pi^{2}} \sqrt{\rho_{C} h / D_{C}}, D_{C}=E_{C} h^{3} / 12\left(1-v_{C}^{2}\right), \\
& k_{g}=K_{g} b^{2} / D_{C}, k_{w}=K_{w} b^{4} / D_{C}
\end{aligned}
$$

According to the data presented in the above-mentioned tables, excellent solution agreements can be observed between the present method and those of the other methods. In this section, we characterize the response of FG-MWCNTs sandwich plate with graded reinforcement volume fractions in the plate's thickness resting on an elastic foundation. The non-dimensional natural frequency, Winkler and shearing layer elastic coefficients are assumed as follows:

$$
\begin{aligned}
& \Omega=\omega \frac{b^{2}}{\pi^{2}} \sqrt{\rho_{i} h / D_{i}}, D_{i}=E_{i} / 12\left(1-v_{i}^{2}\right), \\
& K_{g}=k_{g} b^{2} / D_{i}, K_{w}=k_{w} b^{4} / D_{i}
\end{aligned}
$$

Table 3 Comparison of the first three non-dimensional natural frequency parameters of a simply supported square isotropic plate on the elastic foundation $\left(K_{g}=10\right)$.

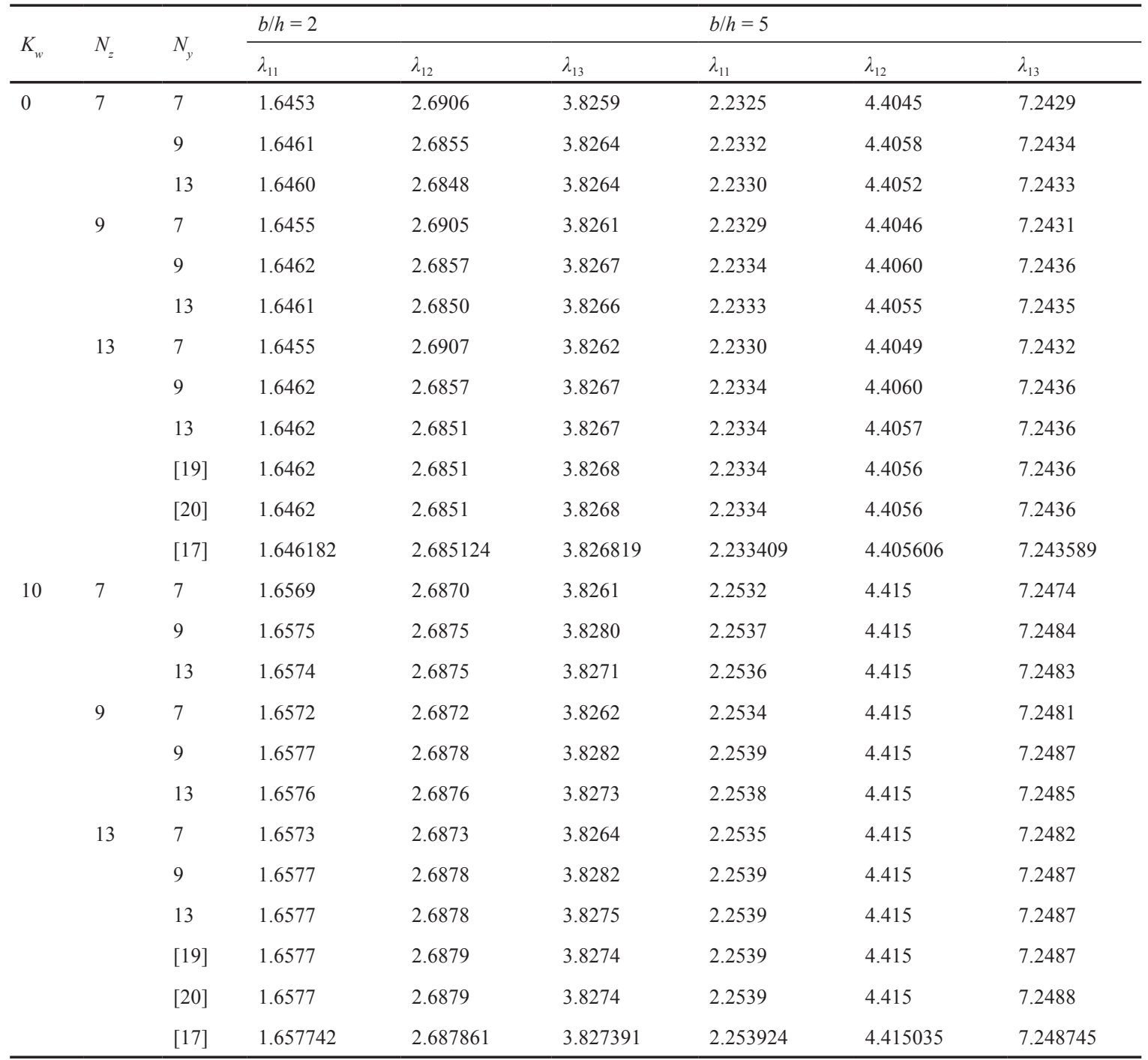



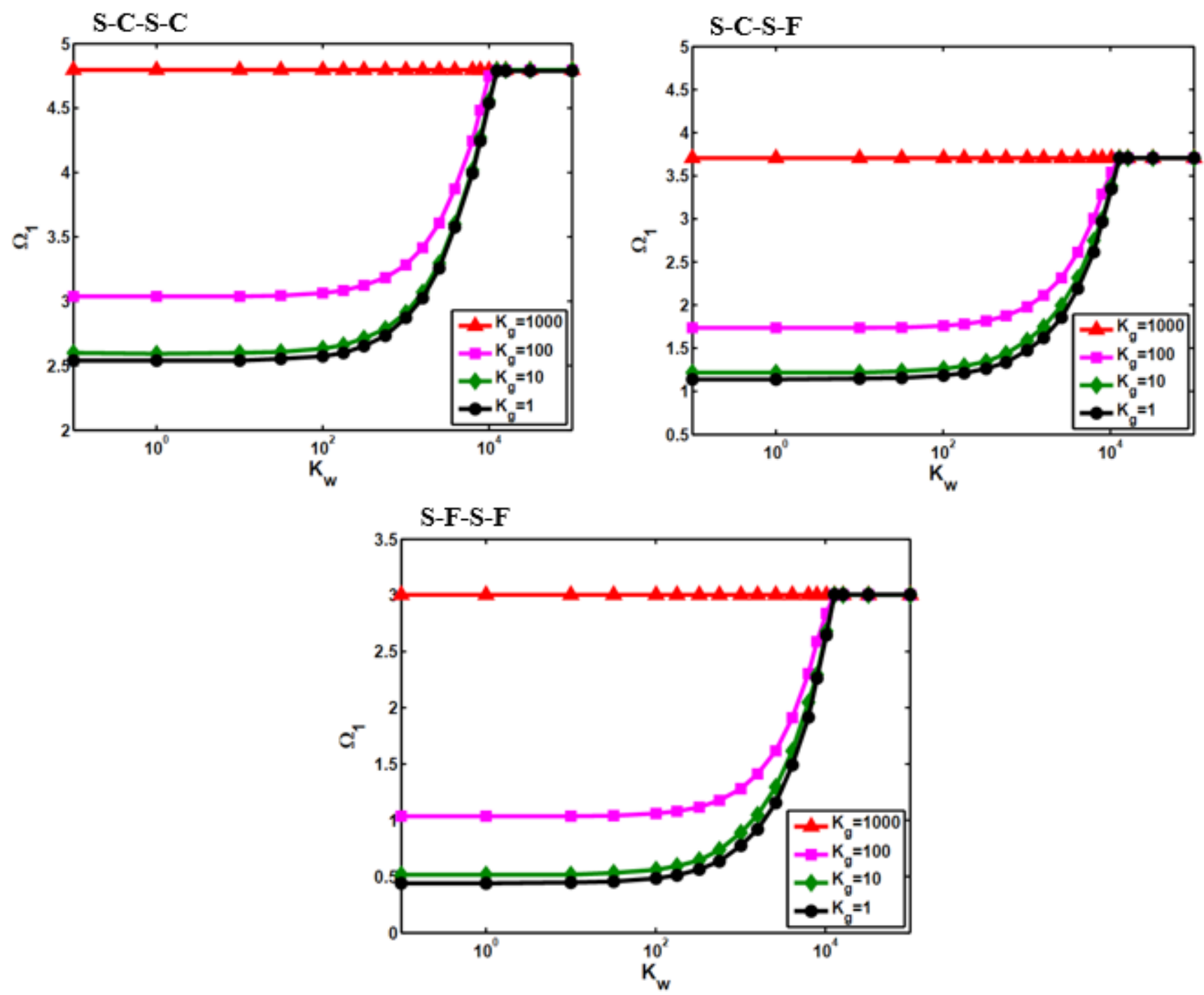

Fig. 4 Variation of the first non-dimensional natural frequency parameter of FG-MWCNT sandwich rectangular plate with Winkler and different shearing layer elastic coefficient for different types of boundary condition $(\mathrm{p}=1, h / b=0.5, a / b=1)$

where $\rho_{i}, E_{i}$ and $v_{i}$ are mechanical properties of are mechanical properties of MWCNT.

The effects of variation of the Winkler elastic coefficient on the first non-dimensional natural frequency parameters of FG-MWCNT sandwich rectangular plate and for different values of shearing layer elastic coefficient and sets of boundary conditions are shown in Fig. 4.

It is clear that in all cases, with increasing the elastic coefficients of foundation, the frequency parameters increase to some limit values.

It is observed for the large values of Winkler elastic coefficient, the shearing layer elastic coefficient has less effect and the results become independent of it.

The influence of shearing layer elastic coefficient on the first non-dimensional natural frequencies for S-C-S-C boundary condition is shown in Fig. 5.

One can see that the Winkler elastic coefficient has little effect on the non-dimensional natural frequencies at different values of shearing layer elastic coefficient.

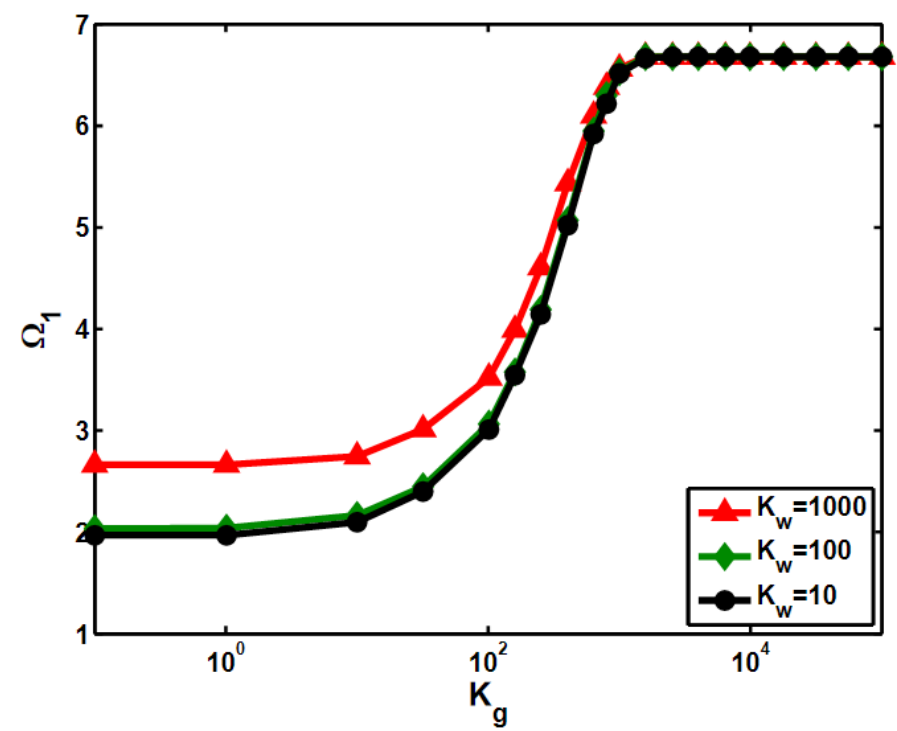

Fig. 5 Variation of the first non-dimensional natural frequency parameters of FG-MWCNT sandwich rectangular plate versus the shearing layer elastic coefficient for different Winkler elastic coefficient and S-C-S-C boundary

condition $(\mathrm{p}=1, h / b=0.5, a / b=1)$ 


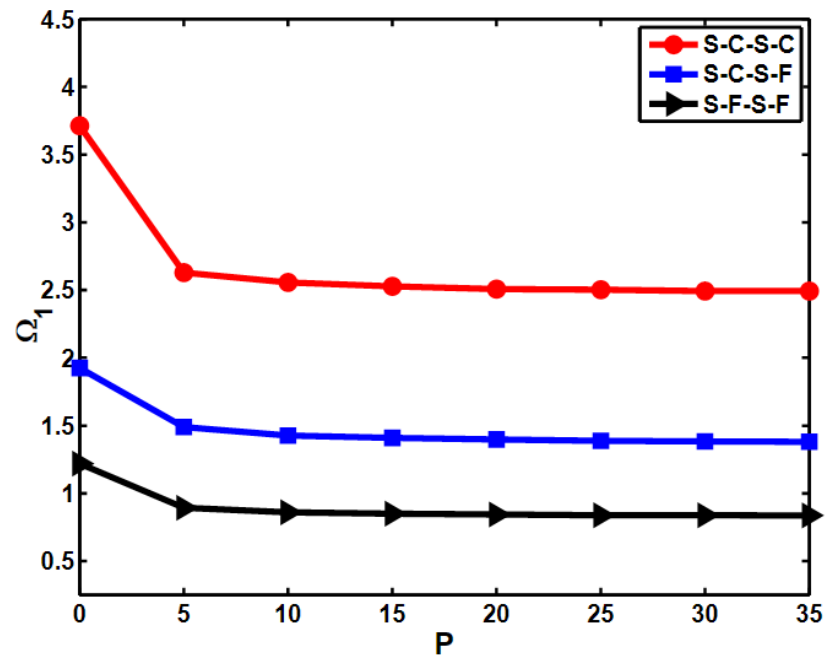

Fig. 6 Variations of the frequency parameters versus " $p$ " for different types of boundary conditions $\left(\mathrm{p}=1, K_{g}=K_{w}=100, a / b=1, h / b=0.5\right)$

It should be noted that this behaviour is also observed at other types of boundary conditions, but, for the sale of brevity, they are not shown here. The influence of constituent volume fractions is studied by varying the volume fractions of MWCNT. This is carried out by varying the power-law exponent " $p$ ".

In Fig. 6, variations of the first non-dimensional natural frequencies versus power-law exponent " $p$ " with respect to different types of boundary conditions for FG-MWCNT sandwich plate is presented.

It is observed that with increasing power-law exponent " $p$ " the first three non-dimensional natural frequencies decrease sharply and then remain almost unaltered for "p" greater than 10 .

As also depicted from Fig. 6, S-C-S-C sandwich plate has the highest, whereas the S-F-S-F one has the lowest non-dimensional natural frequency parameters, it means that the greater supporting rigidity will lead to higher amount of non-dimensional natural frequency parameter. Fig. 7 shows the effect of length-to-width ratio $(a / b)$ on the vibrational behaviour of FG-MWCNT sandwich plates resting on an elastic foundation.

It is seen that for different types of boundary conditions, at first the non-dimensional natural frequency parameter decreases sharply, but, by increasing the length-to-width ratio $(a / b)$ it decreases very slowly and remains somehow unaltered.

\section{Conclusion}

In this research work, free vibration of FG-MWCNT sandwich rectangular plates on a two-parameter elastic foundation is investigated based on three-dimensional theory of elasticity. The main contribution of this work is to present useful results for continuous grading of FG-MWCNT reinforcement in the thickness direction of sandwich plates on two-parameter elastic foundations. The elastic foundation is considered as a Pasternak model with adding a shear layer to the Winkler model. Three complicated equations of motion for the plate under consideration are semi-analytically solved by using 2-D differential

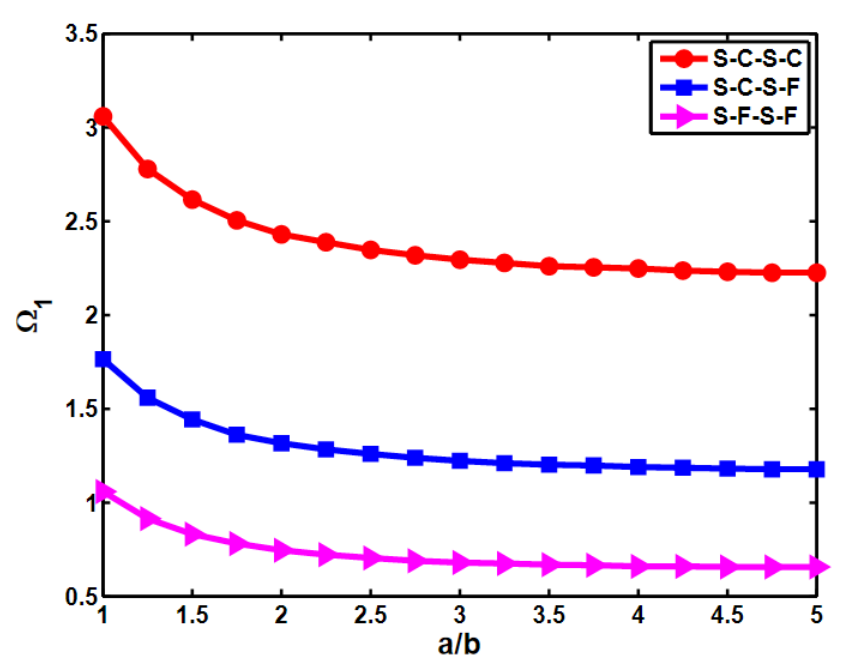

Fig. 7 The effect of length to width ratio of the FG-MWCNT sandwich rectangular plate resting on a two-parameter elastic foundation $\left(\mathrm{p}=1, K_{g}=K_{w}=100, h / b=0.5\right)$

quadrature method. Using the 2-D differential quadrature method in the $\mathrm{y}$ - and $\mathrm{z}$-directions, allows one to deal properly with FG-MWCNT plates with arbitrary thickness distribution of material properties and also to implement the effects of the elastic foundations as a boundary condition on the lower surface of the plate efficiently and in an exact manner.

The fast rate of convergence and accuracy of the method are investigated through the different solved examples. The effects of different geometrical parameters such as the length-to-width ratio, the elastic foundation parameters and boundary conditions on the performance of the natural frequency parameters of the FG-MWCNT sandwich plates are investigated. From numerical illustration, some conclusion can be made as follow:

- The non-dimensional natural frequency parameters converge with increasing Winkler elastic coefficient of the foundation, it should be noted that non-dimensional natural frequency parameters converge at the large values of the Winkler elastic coefficient.

- It is shown that for the large values of shearing layer elastic coefficient; the results become independent of it. It is also shown that with increasing the elastic coefficients of the foundation, the frequency parameters increase to some limit values.

- Results show that for the large values of Winkler elastic coefficient, the shearing layer elastic coefficient has less effect and the results become independent of it.

- It is observed that with increasing power-law exponent " $p$ " the first three non-dimensional natural frequencies decrease sharply and then remain almost unaltered for "p" greater than 10 .

- The results show that for different types of boundary conditions, at first the non-dimensional natural frequency parameter decreases sharply, but, by increasing the length-to-width ratio $(a / b)$ it decreases very slowly and remains somehow unaltered. 


\section{Appendix}

$$
\begin{aligned}
& F_{1 x}=c_{11} \frac{\partial^{2}}{\partial x^{2}}+c_{66} \frac{\partial^{2}}{\partial y^{2}}+\frac{\partial c_{55}}{\partial z} \frac{\partial}{\partial z}+c_{55} \frac{\partial^{2}}{\partial z^{2}}, \\
& F_{1 y}=c_{12} \frac{\partial^{2}}{\partial x \partial y}+c_{66} \frac{\partial^{2}}{\partial y \partial x}, \\
& F_{1 z}=c_{13} \frac{\partial^{2}}{\partial x \partial z}+\frac{\partial c_{55}}{\partial z} \frac{\partial}{\partial x}+c_{55} \frac{\partial^{2}}{\partial z \partial x}, \\
& F_{2 x}=c_{66} \frac{\partial^{2}}{\partial x \partial y}+c_{12} \frac{\partial^{2}}{\partial y \partial x}, \\
& F_{2 y}=c_{66} \frac{\partial^{2}}{\partial x^{2}}+c_{22} \frac{\partial^{2}}{\partial y^{2}}+\frac{\partial c_{44}}{\partial z} \frac{\partial}{\partial z}+c_{44} \frac{\partial^{2}}{\partial z^{2}}, \\
& F_{2 z}=c_{23} \frac{\partial^{2}}{\partial y \partial z}+\frac{\partial c_{44}}{\partial z} \frac{\partial}{\partial y}+c_{44} \frac{\partial^{2}}{\partial z \partial y}, \\
& F_{3 x}=c_{55} \frac{\partial^{2}}{\partial x \partial z}+\frac{\partial c_{13}}{\partial z} \frac{\partial}{\partial x}+c_{13} \frac{\partial^{2}}{\partial z \partial x}, \\
& F_{3 y}=c_{44} \frac{\partial^{2}}{\partial y \partial z}+\frac{\partial c_{23}}{\partial z} \frac{\partial}{\partial y}+c_{23} \frac{\partial^{2}}{\partial z \partial y}, \\
& F_{3 z}=c_{55} \frac{\partial^{2}}{\partial x^{2}}+c_{44} \frac{\partial^{2}}{\partial y^{2}}+\frac{\partial c_{33}}{\partial z} \frac{\partial}{\partial z}+c_{33} \frac{\partial^{2}}{\partial z^{2}}
\end{aligned}
$$

\section{References}

[1] Wagner, H. D., Lourie, O., Feldman, Y. "Stress-induced fragmentation of multiwall carbon nanotubes in a polymer matrix." Applied Physics Letters. 72(2), pp. 188-190. 1997.

https://doi.org/10.1063/1.120680

[2] Fidelus, J. D., Wiesel, E., Gojny, F.H., Schulte, K., Wagner, H. D. "Thermo-mechanical properties of randomly oriented carbon/epoxy nanocomposites." Composites Part A. 36(11), pp. 1555-1561. 2005. https://doi.org/10.1016/j.compositesa.2005.02.006

[3] Ghavamian, A., Rahmandoust, M., Oshner A. "A numerical evaluation of the influence of defects on the elastic modulus of single and multi-walled carbon nanotubes." Computational Materials Science. 62, pp. 110-116. 2012. https://doi.org/10.1016/j.commatsci.2012.05.003

[4] Gojny, F. H., Wichmann, M. H. G., Fiedler, B., Schulte, K. "Influence of different carbon nanotubes on the mechanical properties of epoxy matrix composites-A comparative study." Composites Science Technology. 65(15-16), pp. 2300-2313. 2005.

https://doi.org/10.1016/j.compscitech.2005.04.021

[5] Montazeri, A., Javadpour, J., Khavandi, A., Tcharkhtchi, A., Mohajeri, A. "Mechanical properties of multi-walled carbon nanotube/epoxy composites." Materials \& Design. 31(9), pp. 4202-4208. 2010.

https://doi.org/10.1016/j.matdes.2010.04.018

[6] Yeh, M. K., Tai, N. H., Liu, J. H. "Mechanical behavior of phenolic-based composites reinforced with multi-walled carbon nanotubes." Carbon. 44(1), pp. 1-9. 2006.

https://doi.org/10.1016/j.carbon.2005.07.005

[7] Martone, A., Faiella, G., Antonucci, V., Giordano, M., Zarrelli, M. "The effect of the aspect ratio of carbon nanotubes on their effective reinforcement modulus in an epoxy matrix." Composites Science Technology. 71(8), pp. 1117-1123. 2011.

https://doi.org/10.1016/j.compscitech.2011.04.002

[8] Bellman, R., Casti, J. "Differential quadrature and long term integration." Journal of Mathematical Analysis and Applications. 34(2), pp. 235-238. 1971.

https://doi.org/10.1016/0022-247X(71)90110-7
[9] Tahouneh, V. "Using an equivalent continuum model for 3D dynamic analysis of nanocomposite plates." Steel and Composite Structures, An Int'l Journal. 20(3), pp. 623-649. 2016.

https://doi.org/10.12989/scs.2016.20.3.623

[10] Tahouneh, V., Naei, M. H. "Using Eshelby-Mori-Tanaka scheme for 3D free vibration analysis of sandwich curved panels with functionally graded nanocomposite face sheets and finite length." Polymer Composites. 2016. https://doi.org/10.1002/pc.23929

[11] Tahouneh, V. "Free vibration analysis of bidirectional functionally graded annular plates resting on elastic foundations using differential quadrature method." Structural Engineering and Mechanics, An Int'l Journal. 52(4), pp. 663-686. 2014.

https://doi.org/10.12989/sem.2014.52.4.663

[12] Tahouneh, V., Naei, M. H. "3D free vibration analysis of elastically supported thick nanocomposite curved panels with finite length and different boundary conditions via 2-D GDQ method." Mechanics of Advanced Materials and Structures. 23(10), pp. 1216-1235. 2015. https://doi.org/10.1080/15376494.2015.1068402

[13] Liu, F. L., Liew, K. M. "Free vibration analysis of Mindlin sector plates numerical solutions by differential quadrature method." Computer Methods in Applied Mechanics and Engineering. 177(1-2), pp. 77-92. 1999. https://doi.org/10.1016/S0045-7825(98)00376-4

[14] Liew, K. M., Liu, F. L. "Differential quadrature method for vibration analysis of shear deformable annular sector plates." Journal of Sound and Vibration. 230(2), pp. 335-356. 2000.

https://doi.org/10.1006/jsvi.1999.2623

[15] Wang, X., Wang, Y. "Free vibration analyses of thin sector plates by the new version of differential quadrature, method." Computer Methods in Applied Mechanics and Engineering. 193(36-38), pp. 3957-3971. 2004. https://doi.org/10.1016/j.cma.2004.02.010

[16] Liew, K. M., Han, J. B., Xiao, Z. M., Du, H. "Differential quadrature method for Mindlin plates on Winkler foundation." International Journal of Mechanical Sciences. 38(4), pp. 405-421. 1996. https://doi.org/10.1016/0020-7403(95)00062-3

[17] Yas, M. H., Sobhani, A. B. "Free vibration analysis of continuous grading fiber reinforced plates on elastic foundation." International Journal of Engineering Science. 48(12), pp. 1881-1895. 2010. https://doi.org/10.1016/j.ijengsci.2010.06.015

[18] Matsunaga, H. "Free vibration and stability of functionally graded plates according to a $2 \mathrm{D}$ higher-order deformation theory." Composite Structures. 82, pp. 499-512. 2008.

https://doi.org/10.1016/j.compstruct.2007.01.030

[19] Zhou, D., Cheung, Y. K., Lo, S. H., Au, F. T. K. "Three-dimensional vibration analysis of rectangular thick plates on Pasternak foundation." Numerical Methods in Engineering. 59(10), pp. 1313-1334. 2004. https://doi.org/10.1002/nme.915

[20] Matsunaga, H. "Vibration and stability of thick plates on elastic foundations." Journal of Engineering Mechanics ASCE. 126(1), pp. 27-34. 2000. https://doi.org/10.1061/(ASCE)0733-9399(2000)126:1(27)

[21] Tornabene, F., Viola, E. "Free vibrations of four-parameter functionally Graded parabolic panels and shells of revolution." European Journal of Mechanics - A/Solids. 28(5), pp. 991-1013. 2009.

https://doi.org/10.1016/j.euromechsol.2009.04.005

[22] Viola, E., Tornabene, F. "Free Vibrations of Three Parameter Functionally Graded Parabolic Panels of Revolution." Mechanics Research Communications. 36(5), pp. 587-594. 2009. https://doi.org/10.1016/j.mechrescom.2009.02.001

[23] Shu, C. "Differential quadrature and its application in engineering." Springer, Berlin. 2000. 
[24] Dong, C. Y. "Three-dimensional free vibration analysis of functionally graded annular plates using the Chebyshev-Ritz method." Materials \& Design. 29(8), pp. 1518-1525. 2008.

https://doi.org/10.1016/j.matdes.2008.03.001

[25] Nie, G. J., Zhong, Z. "Semi-analytical solution for three-dimensional vibration of functionally graded circular plates." Computer Methods in Applied Mechanics and Engineering. 196(49-52), pp. 4901-4910. 2007. https://doi.org/10.1016/j.cma.2007.06.028

[26] Nie, G. J., Zhong, Z. "Dynamic analysis of multi-directional functionally graded annular plates." Applied Mathematical Modelling. 34(3), pp. 608616. 2010.

https://doi.org/10.1016/j.apm.2009.06.009

[27] Bakora, A., Tounsi, A. "Thermo-mechanical post-buckling behavior of thick functionally graded plates resting on elastic foundations." Structural Engineering and Mechanics, An Int'l Journal. 56(1), pp. 85-106. 2015.

https://doi.org/10.12989/sem.2015.56.1.085

[28] Kamarian, S., Shakeri, M., Yas, M. H., Bodaghi, M., Pourasghar, A. "Free vibration analysis of functionally graded nanocomposite sandwich beams resting on Pasternak foundation by considering the agglomeration effect of CNTs." Journal of Sandwich Structures and Materials. 17(6), pp. 1-31. 2015.

https://doi.org/10.1177/1099636215590280

[29] Anderson, T. A. "A 3-D elasticity solution for a sandwich composite with functionally graded core subjected to transverse loading by a rigid sphere." Composite Structures. 60(3), pp. 265-274. 2003. https://doi.org/10.1016/S0263-8223(03)00013-8

[30] Kashtalyan, M., Menshykova, M. "Three-dimensional elasticity solution for sandwich panels with a functionally graded core." Composite Structures. 87(1), pp. 36-43. 2009. https://doi.org/10.1016/j.compstruct.2007.12.003

[31] Li, Q., Iu, V., Kou, K. "Three-dimensional vibration analysis of functionally graded material sandwich plates." Journal of Sound and Vibration. 311(1-2), pp. 498-515. 2008. https://doi.org/10.1016/j.jsv.2007.09.018

[32] Zenkour, A. "A comprehensive analysis of functionally graded sandwich plates, Part 1-Deflection and stresses." International Journal of Solids and Structures. 42(18-19), pp. 5224-5242. 2005. https://doi.org/10.1016/j.ijsolstr.2005.02.015

[33] Zenkour, A. "A comprehensive analysis of functionally graded sandwich plates: Part 2-Buckling and free vibration." International Journal of Solids and Structures. 42(18-19), pp. 5243-5258. 2005.

https://doi.org/10.1016/j.ijsolstr.2005.02.016
[34] Sharma, K., Marin, M. "Effect of distinct conductive an thermodynamic temperatures on the reflection of plane waves in micropolar elastic halfspace." U.P.B. Scientific Bulletin, Series A-Applied Mathematics and Physics. 75(2), pp. 121-132. 2013.

[35] Marin, M. "On weak solutions in elasticity of dipolar bodies with voids." Journal of Computational and Applied Mathematics. 82(1-2), pp. 291297. 1997.

https://doi.org/10.1016/S0377-0427(97)00047-2

[36] Marin, M. "Harmonic vibrations in thermoelasticity of microstretch materials." Journal of Vibration and Acoustics, ASME. 132(4), pp. 501506. 2010.

https://doi.org/10.1115/1.4000971

[37] Marin, M., Agarwal, R. P., Mahmoud, S. R. "Modeling a Microstretch Thermoelastic Body with Two Temperatures." Abstract and Applied Analysis. 2013, pp. 1-7. 2013. https://doi.org/10.1155/2013/583464

[38] Heshmati, M., Yas, M. H. "Vibrations of non-uniform functionally graded MWCNTs-polystyrene nanocomposite beams under action of moving load." Material \& Design. 46, pp. 206-218. 2013. https://doi.org/10.1016/j.matdes.2012.10.002

[39] Farid, M., Zahedinejad, P., Malekzadeh, P. "Three dimensional temperature dependent free vibration analysis of functionally graded material curved panels resting on two parameter elastic foundation using a hybrid semi-analytic, differential quadrature method." Materials \& Design. 31(1), pp. 2-13. 2010.

https://doi.org/10.1016/j.matdes.2009.07.025

[40] Jam, J. E., Pourasghar, A., Kamarian, S. "The effect of the aspect ratio and waviness of CNTs on the vibrational behavior of functionally graded nanocomposite cylindrical panels." Polymer Composites. 33(11), pp. 2036-2044. 2012. https://doi.org/10.1002/pc.22346

[41] Halpin, J. C., Tsai, S. W. "Effects of environmental factors on composite materials." AFML-TR-67-423. 1969.

[42] Halpin, J. C., Kardos, J. L. "The Halpin-Tsai equations: A review." Polymer Engineering \& Science. 16(5), pp. 344-352. 1976. https://doi.org/10.1002/pen.760160512

[43] Pelletier Jacob, L., Vel Senthil, S. "An exact solution for the steady state thermo elastic response of functionally graded orthotropic cylindrical shells." International Journal of Solid Structures. 43(5), pp. 1131-1158. 2006. https://doi.org/10.1016/j.ijsolstr.2005.03.079 